\title{
Dense middle cerebral artery sign: an indicator of poor outcome in middle cerebral artery area infarction
}

\author{
JYRKI LAUNES, ${ }^{*}$ LEENA KETONEN $\dagger$ \\ From the Departments of Neurology* and Radiology, Division of Neuroradiology, $\dagger$ Helsinki University Central \\ Hospital, Finland
}

SUMMARY Thirteen patients with a dense appearance of the horizontal part of the middle cerebral artery (MCA) “dense middle cerebral artery sign" in CT scans taken within 24 hours after onset of ischaemic stroke had considerably poorer prognosis than controls with stroke, but without the sign. A hyperdense appearance of the MCA is known to be associated with thromboembolism, but dense middle cerebral artery sign is also an early warning of a large infarction, brain oedema and poor prognosis in infarction in the MCA area.

In a period of 2 weeks, we saw three cases of MCA infarction with the dense middle cerebral artery sign in CT scans taken during the first 24 hours after onset of stroke. Each patient died within a week from the first symptoms. We investigated the possible predictive value of the dense middle cerebral artery sign, because hypodense lesions showing the area of infarction are likely not to be seen on CT during this time.

The volume of infarction seen on CT scans is a prognostic sign. ${ }^{1}$ Unfortunately, CT first becomes positive 8-24 hours after the onset of ischaemic stroke symptoms, ${ }^{2}$ thus not allowing immediate assessment of patients' prognosis. The dense appearance of the MCA on CT was first reported by Gacs et al in seven patients. They correlated the finding with thromboembolism, but not to clinical outcome. We now report that this early-phase CT finding indicates a poor clinical outcome in infarction in the MCA area.

\section{Patients and methods}

We searched for the presence of the dense middle cerebral artery sign in CT scans taken during the first 24 hours after onset of stroke in patients with acute ischaemic hemiparesis during a period of one year. All the scans were seen by a neuroradiologist (LK).

Patients and controls were included in the study according

Address for reprint requests: Dr Jyrki Launes, Haartmaninkatu 4, 00290 Helsinki 29, Finland.

Received 9 January 1987 and in revised form 14 March 1987. Accepted 16 May 1987. to the following criteria: (1) CT was performed no longer than 24 hours after onset of symptoms; (2) There were neurological signs and symptoms or radiological evidence of MCA vascular area infarction at the time of admission; (3) There were no signs of progressive stroke; (4) The time of onset of symptoms was accurately known. Patients with a dense appearance of the horizontal part of the MCA contralateral to hemiparesis were included in the dense middle cerebral artery sign group, provided that no blood was seen in the subarachnoid space and the sign was not bilateral. Fifty cases fulfilled all the criteria (13 sign positive and 37 sign negative).

Clinical status at the time of admission and discharge was assessed in a retrospective view from the patients' charts. The patients' and controls' condition at the time of admission was scored into five categories: (1) Only minor neurological symptoms, such as minor hemiparesis, dysphasia, or minimal disturbance of coordination. (2) Moderate hemiparesis or dysphasia. (3) Hemiparesis with some movement in upper or lower limb. (4) Hemiparalysis. (5) Hemiparesis and impaired consciousness. The clinical outcome was scored in five categories. (1) Fully independent in activities of daily living (ADL). (2) Requires minor assistance in ADL. (3) Requires constant assistance in ADL. (4) Totally dependent, requires hospitalisation. (5) Died. Subjects having an outcome scored to grade 3-5 were considered to have a poor outcome. All sign positive patients had the clinical score 3-5. Twenty one control patients whose clinical score was 3-5 were included in order to build a control group of those who could not be separated from the patients on clinical grounds.

CT was performed without contrast enhancement. CT was repeated on three patients $(30,48$ and 50 hours after the initial scanning). Siemens Somatom 2 or General Electric 8800 CT scanners were used. Statistical significance of 

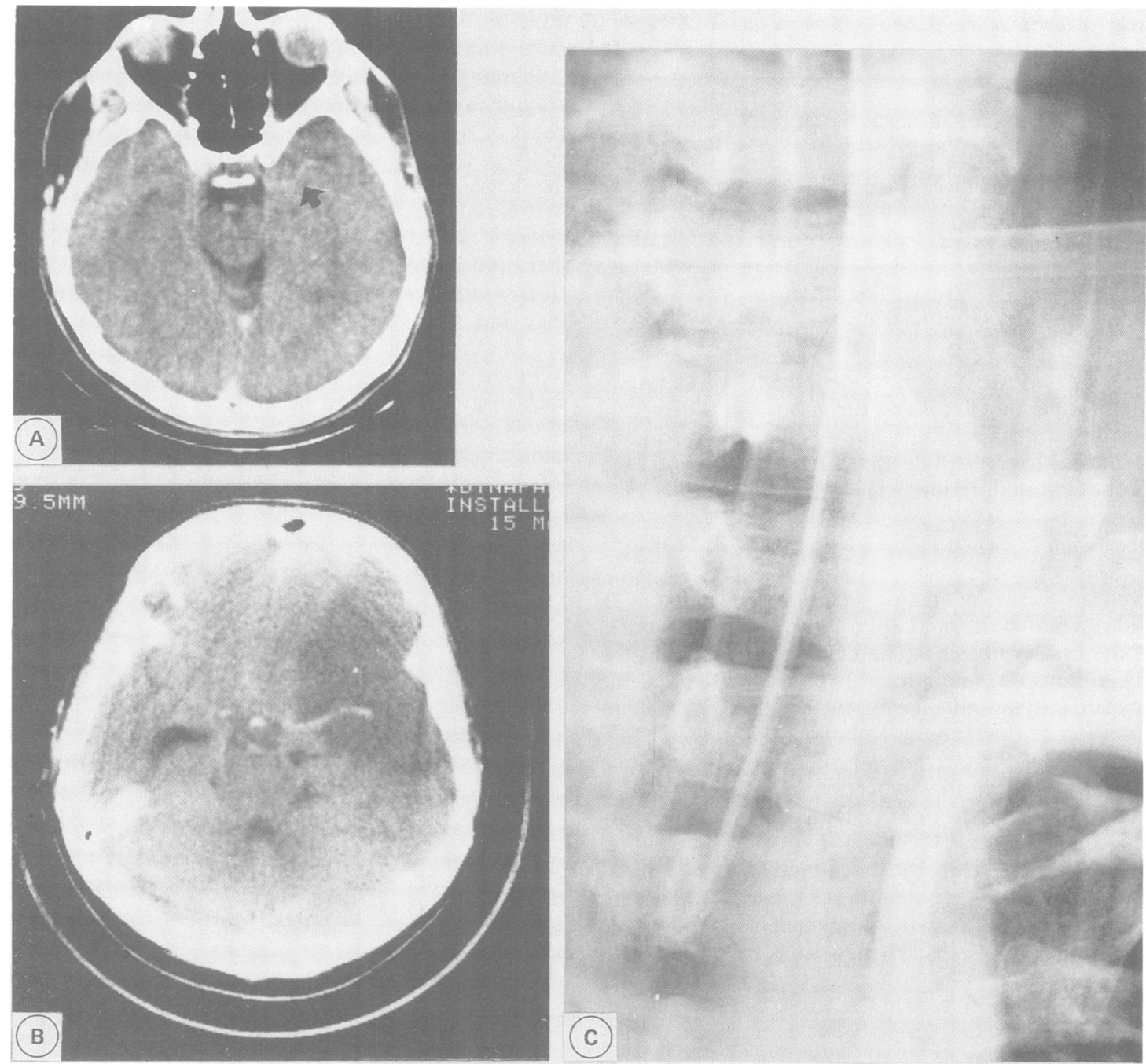

Fig (A) Dense middle cerebral artery sign in a male aged $36 y$. The horizontal part of the left middle cerebral artery (MCA) appears dense. The contralateral MCA is normal. No signs of ischaemic brain infarction are yet visible. Selective left carotid angiography revealed a thrombus in the common carotid artery extending to the internal carotid artery. The patient died four days after first symptoms due to elevated intracranial pressure. At necropsy, an atheromatous plaque was found in the bifurcation of internal carotid artery with secondary thrombosis extending from common carotid artery to distal MCA. (B) Follow-up CT two days later demonstrates a large left MCA infarction and a dense horizontal part of the MCA.

differences in outcome was tested with Mantel-Haenszel chisquare test. Statistical significance of differences in age and time between onset of symptoms and CT were tested with Student's $t$ test.

\section{Results}

CT was performed $7.8 \pm 2.5$ hours in patients and $12.1 \pm 7.6$ hours in controls after the onset of symptoms $(\mathrm{p}<0.06)$. Ten of 13 patients with the dense middle cerebral artery sign died, while only two of 21 controls died during the stay in hospital $(p<0.001)$ (table). The clinical outcome was grade 3,4 or 5 in 12 of 13 patients and 10 of 21 controls $(P<0.05)$. Nine of 13 patients with the dense middle cerebral artery sign, and four of 21 controls had symptoms of intracranial hypertension at the time of admission ( $p<$ 0.05 ). No differences between the patients' ages or side of the hemiparesis were found. The dense middle cerebral artery sign had disappeared in $2 / 3$ repeat scans. Necropsy was performed in six cases. In one 
Table Clinical data of patients with dense middle cerebral artery sign and controls

\begin{tabular}{lcc}
\hline & Dense-media & Controls \\
\hline $\mathbf{N}$ & 13 & 21 \\
Mean age (yr) & $55 \cdot 7$ & $54 \cdot 5$ \\
Hours between CT scan and first symptom & $7 \cdot 8$ & $12 \cdot 1$ \\
Died & 10 & $2 \ddagger$ \\
Poor outcome & 12 & $10^{*}$ \\
Intracranial hypertension during first $24 \mathrm{~h}$ & 9 & $4^{*}$
\end{tabular}

${ }^{*} \mathrm{p}<0.05$

$\pm \mathrm{p}<0.001$

In Mantel-Haenszel chi square or Student's $t$ test.

case a massive embolism to the left MCA was found. In the remaining five cases, thrombosis of the internal carotid artery, reaching up to the corresponding MCA was found. All deaths in our cases were caused by the brain infarction.

\section{Discussion}

Even in cases of major cerebral infarction, CT findings may be normal up to 24 hours after the onset of the first symptoms. The dense appearance of the MCA, however, may probably be seen as soon as the ischaemic symptoms have started, as it is a manifestation of the thromboembolic event.

Lesions of the internal capsule, right parietal lobe or a mass effect in connection with major stroke symptoms, relate to poor outcome. ${ }^{4}$ In a recent survey from our country, $30 \%$ of patients with cerebral infarction died during the first 3 months after onset of illness, but also other than brain-related deaths were recorded in the study. ${ }^{5}$ In another survey based on the same series, $65 \%$ of surviving patients were independent in ADL after 12 months. ${ }^{6}$ The prognosis of surviving a MCA occlusion in the acute stage was $20 \%$ in patients with angiographically demonstrated disease. ${ }^{7}$ In our series, $77 \%$ of patients with dense middle cerebral artery sign died during the stay in hospital, while only $10 \%$ of the patients in the control group died. Only $8 \%$ of patients, but no less than $50 \%$ of controls had good outcome (grade 1 or 2). The time between onset of the symptoms and the CT was somewhat shorter in patients than in controls $(7 \cdot 4$ vs 13.8 hours; $p<0.01$ ).

Patients more often had signs of elevated intracranial pressure during the first 24 hours after the onset of the first symptoms, which was the only clinical sign that predicted poor outcome in addition to the dense middle cerebral artery sign.

It is not clear how soon the dense middle cerebral artery sign disappears. It was not seen in two repeat scans ( 30 and 50 hours after the first scans), but was clearly visible in the follow-up CT of the illustrated case (fig).

The outcome of our study groups differs from that reported on literature, ${ }^{7}$ whereas the prognosis in our control group seems to be more in accordance with that reported in our country. ${ }^{5}$ The presence of the dense middle cerebral artery sign suggests a massive infarction with poor clinical outcome.

The authors express their sincere gratitude to Ms Kirsti Nikonen for her invaluable help in the collection of data.

\section{References}

1 Tagaki S, Shinohara Y. Internal carotid occlusion: volume of cerebral infarction, clinical findings, and prognosis. Stroke 1981;12:835-9.

2 Houser OW, Campbell JK, Baker HL, Sundt TS. Radiologic evaluation of ischaemic cerebrovascular syndromes with emphasis on computed tomography. Radiol Clin N Am 1982;20:123-42.

3 Gacs G, Fox AJ, Barnett HJM, Vinuela F. CT visualisation of intracranial arterial thromboembolism. Stroke 1983;14:756-62.

4 Valdimarsson E, Bergvall U, Samuelson K. Prognostic significance of cerebral computed tomography results in supratentorial infarction. Acta Neurol Scand 1982;65:133-45.

5 Kotila M. Declining incidence and mortality of stroke? Stroke 1984;15:255-9.

6 Kotila M, Waltimo O, Niemi M-L, Laaksonen R, Lempinen $M$. The profile of recovery from stroke and factors influencing outcome. Stroke 1985;15:1039-44.

7 Moulin DE, Lo R, Chiang J, Barnett HJM. Prognosis in middle cerebral artery occlusion. Stroke 1985;16: 282-4. 